\title{
Increasing and Sustaining Participation to Support and Foster Social Creativity
}

\author{
Holger Dick, Hal Eden, and Gerhard Fischer \\ Center for LifeLong Learning \& Design $\left(\mathrm{L}^{3} \mathrm{D}\right)$ \\ University of Colorado \\ Boulder, CO 80309-0430 USA \\ \{holger.dick, haleden, gerhard\}@colorado.edu
}

\begin{abstract}
The rise in social computing has facilitated a shift from consumer cultures to cultures of participation. These developments represent unique and fundamental opportunities and challenges for social creativity.

The CreativeIT Wiki project represents an effort to explore and build a socio-technical environment for members of the emerging research community interested in creativity and information technology.
\end{abstract}

\section{Keywords}

cultures of participation, social creativity, democratizing creativity, next-generation wikis

\section{INTRODUCTION}

Most pressing and important problems of today's world are complex and ill-defined systemic problems that make collaboration among people from different disciplines a necessity. Socio-technical environments [Mumford, 2000] that unleash and democratize creativity [von Hippel, 2005] are needed to achieve these goals. Following a design-based approach to research, we are creating such a socio-technical environment for the community surrounding the NSF CreativeIT program.

\section{CULTURES OF PARTICIPATION AND SOCIAL CREATIVITY}

Cultures of Participation. The emerging and expanding cyberinfrastructure and Web 2.0 architectures have begun to support a social or participatory Web, representing a fundamental shift from a consumer culture (passive consumption of finished goods produced by others) to a culture of participation (actively participating in personally meaningful activities). However, deep and enduring changes are not just technological-they result from changes in human behavior and social organizations, requiring the cultivation of socio-technical environments through design, adoption, appropriation, and adaptation to the needs of the participants.

Social Creativity. Individual and social creativity can and need to complement each other. An individual's skills and experience are critical and in complex design problems the different knowledge, expertise, and perspectives that exist among individuals provides opportunities to collaborate toward more creative and sustainable solutions [Fischer et al., 2005]. The same is true for the collaboration between the creative practices and technology [National-Research-Council, 2003] with results transcending the isolated effort of a single artist or computer scientist [Candy \& Edmonds, 2002].

\section{FIRST GENERATION ENVIRONMENT: CreativeIT-WiKl-1}

The underlying goal of this project was to create an online community around the emerging field of Creativity and IT. To achieve this goal, the original vision of this project was to create environments that transcend existing wikis to permit users to become creative inside the environment.

This research effort was engaged in examining limitations of current environments compared with what is needed to support creative practices; exploring support for specific media such as mind maps, videos, anecdotes, and stories; exploring varied "modes" of interacting with such an environment; and utilizing new paradigms (such as the emerging Web 2.0 framework) for developing systems that are open and extensible.

Our first prototype CREATIVEIT-WIKI-1 (http://swiki. cs.colorado.edu/CreativeIT/) did have some minimal degree of success, with the creation of 290 pages, 80 literature/resources references contributed by community members, the ASU workshop proceedings published as part of the wiki, a gallery of project exemplars with an interface to support addition of new material, community member profiles, and hosting over 100 registrants since its inception.

While the prototype had some success as a content management system-marked by the creation of 290 pages, 80 literature/resources references contributed by community members, the ASU workshop proceedings published as part of the wiki, a gallery of project exemplars, and hosting over 100 registrants since its inception-it fell short in creating and fostering an active community. The perceived value and benefits to participate needed to be substantially increased to make participation attractive and to overcome media and 
attention competition. The prototype did not reach the "tipping point" [Gladwell, 2000] of broad-based participation.

\section{SECOND GENERATION ENVIRONMENT: CreativeIT-WIKI-2}

The objective for the second generation, CREATIVEITWIKI-2, was to find ways to engage the community by supporting processes and activities surrounding the creation of content itself. Users should be encouraged to find, create, explore, and participate in communities of interests. To do this, participants need to be able to find out who other participants are, what their interests are, what pages they contribute to [Kittur et al., 2008.], what community activities are happening, and to communicate with each other seamlessly.

\section{Design Criteria of CREATIVEIT-WIKI-2 for Increasing and} Sustaining Participation

Based on our experience, assessment, and feedback from the participating community, combined with insights and analysis of other research efforts, we focused our objectives on:

- Increasing participation by improved utility;

- Supporting visualizations of community relationships;

- Motivating participants to visit regularly;

- Supporting and encouraging the development of examples, models, and tutorials;

- Supporting direct peer-to-peer communication between participants inside the wiki;

- Fostering a community of committed contributors; and

- Supporting migration paths for contributor involvement.

We selected XWIKI (http://www.xwiki.org) as our new substrate as it (1) leverages a broad, active wiki developer community; (2) has an open architecture, an open development process, and support for end-user development; and (3) provides strong support for "Web 2.0 " approaches.

\section{Description of CREATIVEIT-WIKI-2}

We have prototyped new developments and changed certain social approaches and behaviors to test our hypotheses.

To evaluate their effectiveness, we needed a setting that allowed us to get more and quicker feedback. As a result, we tested the new wiki as a course information environment for classes at the University of Colorado (see: http://xwiki.cs.colorado.edu/bin/view/DCNM2009/) with promising results. In brief, we have tested enhanced user profiles, templates for contributions, support mechanisms for peer-to-peer communication as well as broad discussions, and new sophisticated awareness tools (for detailed descriptions of the Wiki, a demo, and results see: $\underline{\text { ttp://13dwiki.cs.colorado.edu/bin/view/NGW/) }}$

\section{ACKNOWLEDGEMENTS}

The authors thank our $L^{3} \mathrm{D}$ colleagues and other CreativeIT researchers who have participate in the wiki.

The research was supported in part by grants from the National Science Foundation including: (a) IIS-0613638, (b) IIS-0709304 and (c) IIS-0843720

\section{REFERENCES}

1. Candy, L. \& Edmonds, E. A. (2002) Explorations in Art and Technology, Springer-Verlag, London.

2. dePaula, R. (2004) The Construction of Usefulness: How Users and Context Create Meaning with a Social Networking System, Ph.D. Dissertation, University of Colorado at Boulder.

3. dePaula, R., Fischer, G., \& Ostwald, J. (2001) "Courses as Seeds: Expectations and Realities." In P. Dillenbourg, A. Eurelings, \& K. Hakkarainen (Eds.), Proceedings of the European Conference on Computer-Supported Collaborative Learning, Maastricht, Netherlands, pp. 494-501.

4. Fischer, G., Giaccardi, E., Eden, H., Sugimoto, M., \& Ye, Y. (2005) "Beyond Binary Choices: Integrating Individual and Social Creativity," International Journal of Human-Computer Studies (IJHCS) Special Issue on Computer Support for Creativity (E.A. Edmonds \& L. Candy, Eds.), 63(4-5), pp. 482-512.

5. Fischer, G. \& Ostwald, J. (2002) "Seeding, Evolutionary Growth, and Reseeding: Enriching Participatory Design with Informed Participation," Proceedings of the Participatory Design Conference (PDC'02), Malmö University, Sweden, pp. 135-143.

6. Gladwell, M. (2000) The Tipping Point: How Little Things Can Make a Big Difference, Back Bay Books, New York, NY.

7. Kittur, A., Suh, B., \& Chi, E. H. (2008.) "Can You Ever Trust a Wiki? Impacting Perceived Trustworthiness in Wikipeda." In Proceedings of the Acm 2008 Conference on Computer Supported Cooperative Work, ACM, New York, NY, pp. 477480.

8. Mumford, E. (2000, "A Socio-Technical Approach to Systems Design," Requirements Engineering,, pp. 5977.

9. National-Research-Council (2003) Beyond Productivity: Information Technology, Innovation, and Creativity, National Academy Press, Washington, DC.

10.von Hippel, E. (2005) Democratizing Innovation, MIT Press, Cambridge, MA. 